Review Article

\title{
A Further Study on Multiperiod Health Diagnostics Methodology under a Single-Valued Neutrosophic Set
}

\author{
Jason Chih-sheng Chou, ${ }^{1}$ Yi-Fong Lin $\mathbb{D}^{2},{ }^{2}$ and Scott Shu-Cheng Lin ${ }^{3}{ }^{3}$ \\ ${ }^{1}$ The MITRE Corporation, McLean, VA 22102, USA \\ ${ }^{2}$ Department of Fashion Industry Management, Hsing Wu University, Taiwan \\ ${ }^{3}$ Department of Hotel Management, Lee-Ming Institute of Technology, Taiwan \\ Correspondence should be addressed to Yi-Fong Lin; daniellyf@mail.lit.edu.tw
}

Received 30 March 2020; Accepted 6 July 2020; Published 1 August 2020

Academic Editor: Markos G. Tsipouras

Copyright ( 2020 Jason Chih-sheng Chou et al. This is an open access article distributed under the Creative Commons Attribution License, which permits unrestricted use, distribution, and reproduction in any medium, provided the original work is properly cited.

\begin{abstract}
Employing the concept and function of tangency with similarity measures and counterpart distances for reliable medical consultations has been extensively studied in the past decades and results in lots of isomorphic measures for application. We compared the majority of such isomorphic measures proposed by various researchers and classified them into (a) maximum norm and (b) one-norm categories. Moreover, we found that previous researchers used monotonic functions to transform an identity function and resulted in complicated expressions. In this study, we provide a theoretical foundation to explain the isomorphic nature of a newer measure proposed by the following research paper against its studied existing one in deriving the same pattern recognition results. Specifically, this study initially proposes two similarity measures using maximum norm, arithmetic mean, and aggregation operators and followed by a detailed discussion on their mathematical characteristics. Subsequently, a simplified version of such measures is presented for easy application. This study completely covers two previous methods to point out that the complex approaches used were unnecessary. The findings will help physicians, patients, and their family members to obtain a proper medical diagnosis during multiple examinations.
\end{abstract}

\section{Introduction}

With the current state of medical consultations, a patient may present with several different inclinations of diseases, especially for multilevel diagnosis. This poses a critical question on how can a physician provide a reliable conclusion for the patient's health status and extend to the patient and his or her family members. Zadeh [1] pioneered the development of fuzzy sets (FSs) to deal with complex, uncertain, vague, and incomplete problems in the physical world. There are many important extensions of the FSs such as the neutrosophic sets (NSs) that were proposed by Smarandache [2]. However, NSs pose to be difficult in actual applications such that Wang et al. [3, 4] developed single-valued neutrosophic sets (SVNSs) to provide a simplification of NSs. SVNSs have three membership functions: truth, indeterminacy, and falsity, which give sufficient information to analyze a patient's condition and will be used to construct a systematic approach to determine the most probable disease. Many papers studied SVNSs: for example, Ye [5] constructed an operator using weighted averages under a hesitant fuzzy element (HFE) environment and a single-valued neutrosophic set. Another study is Ye [6] to consider similarity measures with the cosine function, and then $\mathrm{Ye}$ and $\mathrm{Fu}$ [7] referred to the similarity measures in Ye [6]. Ninety-three papers have cited Ye [6] in their references. We list them in the following: Peng and Dai [8], Ye [9], Biswas et al. [10], Broumi et al. [11], Chatterjee et al. [12, 13], Cui and Ye [14], Fan [15], Fan et al. [16], Fu et al. [17], Gou and Wang [18], Jha et al. [19, 20], Li et al. [2123], Liu and Luo [24], Luo et al. [25], Nancy and Garg [26], Nguyen et al. [27], Peng [28], Peng and Dai [29], Rajangam and Annamalai [30], Şahin [31], Shao et al. [32], Shi and Yuan [33], Tian et al. [34], Wang et al. [35], Wei and Zhang [36], Xie et al. [37], Ye [38], Zhai et al. [39], Zhang et al. [40], Zhao et al. [41], Akram et al. [42], Ali et al. [43], Cai and Yang [44], Fan et al. [45], Garg and Nancy [46], Guan et al. 
[47], Karaaslan and Hayat [48], Khanet al. [49], Küçük and Şahin [50], Liu [51], Liu et al. [52], Liu et al. [53], Liu and You [54], Mondal et al. [55-57], Ngan et al. [58], Peng et al. [59], Pramanik et al. [60, 61], Şahin [62], Singh [63], Tian et al. [64], Tu et al. [65], Wei and Wei [66], Wu et al. [67], Wu et al. [68], Xiong and Cheng [69], Ye [70], Zeming et al. [71], Zhang et al. [72], Chen and Ye [73], Deli and Şubaş [74], Fan and Ye [75], Fu and Ye [76], Hu et al. [77], Lu and Ye [78], Ma et al. [79], Peng et al. [80-82], Pramanik et al. [83], Singh [84], Thanh et al. [85], Tian et al. [86], Wang and Zhang [87], Xu et al. [88], Zhou and Abdel Wahab [89], Chou [90], Liu and Tang [91], Meng et al. [92], Nancy and Garg [93], Shi [94], Son [95], Ye [96], Ye and Fu [7], Zhang et al. [97], Zhang et al. [98], and Zhang et al. [99]. Among those 93 papers, 92 papers only mentioned Ye [6] in their introduction, and then they concentrated on their new similarity measures and applied their new similarity measures to solve real application problems. Only one paper, Ye and $\mathrm{Fu}$ [7] have written down the similarity measures proposed by Ye [6]. However, Ye and Fu [7] did not provide any discussion for the similarity measures proposed by $\mathrm{Ye}$ [6] with cosine functions. Instead, $\mathrm{Ye}$ and $\mathrm{Fu}$ [7] developed new similarity measures with tangent functions. Hence, Ye and $\mathrm{Fu}$ [7] developed two tangent similarity measures and subsequently defined aggregation operators for multiperiod medical diagnosis. There are 49 papers that have cited Ye and $\mathrm{Fu}$ [7] in their references. We list them in the following: Liu et al. [100], Peng and Dai [8], Awang et al. [101], Chatterjee et al. [12, 13], Cui and Ye [14], Fu et al. [17], Gou and Wang [18], Hu et al. [102], Li et al. [21], Long et al. [103], Luo et al. [25], Nancy and Garg [26], Nguyen et al. [27], Rajangam and Annamalai [30], Ren et al. [104], Shen et al. [105], Shi and Yuan [33], Smith [106], Xie et al. [37], Akram et al. [107], Ali et al. [43], Hu et al. [108], Ji et al. [109-111], Karaaslan [112], Karaaslan and Hayat [48], Khan et al. [113], Liu [51], Mondal et al. [55-57], Naz et al. [114], Peng and Dai [115], Tu et al. [65], Uluçay et al. [116], Wu et al. [67], Ye [70], Ashraf et al. [117], Chen and Ye [73], Fu and Ye [76], Guan et al. [118], Guo et al. [119], Hu et al. [77], Lu and Ye [78], Naz et al. [120], Peng and Dai [121], and Thanh et al. [85]. We have examined those 49 papers to find out that they only review $\mathrm{Ye}$ and $\mathrm{Fu}$ [7] in their literature study without any further discussions concerning $\mathrm{Ye}$ and $\mathrm{Fu}$ [7]. There are many similarity measures developed by researchers. However, there are only a few studies that conduct comprehensive examinations for previously published papers. For example, Julian et al. [122] improved three questionable results of Mitchell [123]. Deng and Chao [124] and Tung et al. [125] further revised two questionable findings of Julian et al. [122]. We follow this trend to examine the findings of Ye [6] and Ye and Fu [7] with a detailed analysis.

Prior studies generally repeated in proposing a newer similarity measure and been evaluated against an existing one (i.e., a studied measure) in deriving the same pattern recognition results for medical diagnosis problems. Thus, they result in lots of isomorphic measures. Many researchers as mentioned above claimed that their new similarity measures are supported by existing ones. We took a detailed examination for some similarity measures and found that their coun- terpart distances (that is, similarity measure plus distance is the unity) can be classified into (a) maximum norm and (b) one-norm categories. Additionally, many researchers used monotonic functions to transform the identity function (that is, $f(x)=x$ ) into complicated expressions. These review comments trigger us to study the similarity measures and point out that they are isomorphic when compared to those two categories. Hence, we provide a theoretical foundation to explain why a newly proposed measure by the following paper will derive the same results as its studied one (i.e., an existing measure).

Initially, we will use a simple example to explain the isomorphic property of this research for ordinary readers. We concentrate on $\mathrm{Ye} \mathrm{[6]} \mathrm{and} \mathrm{Ye}$ and $\mathrm{Fu}$ [7], and they are representing an existing measure (i.e., Ye [6]) and the following measure (i.e., Ye and $\mathrm{Fu}$ [7]). Ye [6] used the cosine function, and $\mathrm{Ye}$ and $\mathrm{Fu}$ [7] applied the tangent function, where cosine is a strictly decreasing function, and tangent is a strictly increasing function. The isomorphic property can be understood by assuming $1>\operatorname{dist}(a, x)>\operatorname{dist}(a, y)$, then

$$
\operatorname{Sim}(a, x)<\operatorname{Sim}(a, y)
$$

The strictly decreasing property of cosine implies cos ( dist $(a, x))<\cos (\operatorname{dist}(a, y))$. In this example, Ye [6] would create a new similarity measure as

$$
\operatorname{Sim}_{[6]}(a, x)=\cos (\operatorname{dist}(a, x)) \text {, }
$$

such that

$$
\operatorname{Sim}_{[6]}(a, x)<\operatorname{Sim}_{[6]}(a, y)
$$

In fact, the similarity measure proposed by Ye [6] preserves the original ordering of Equation (1). The strictly increasing property of tangent implies $\tan (\operatorname{dist}(a, x))>\tan$ (dist $(a, y))$. As an example of the following study, Ye and $\mathrm{Fu}$ [7] would create a new similarity measure as

$$
\operatorname{Sim}_{[7]}(a, x)=1-\tan (\operatorname{dist}(a, x)),
$$

such that

$$
\operatorname{Sim}_{[7]}(a, x)<\operatorname{Sim}_{[7]}(a, y)
$$

The new similarity measure proposed by Ye and Fu [7] preserves the original ordering of Equation (1) as well. In Ye and Fu [7], they claimed that they used the tangent function to develop a new similarity measure. We point out that Ye and Fu [7] applied "one minus tangent function" to create a new similarity measure. Based on the isomorphic property, $\mathrm{Ye} \mathrm{[6]} \mathrm{and} \mathrm{Ye} \mathrm{and} \mathrm{Fu} \mathrm{[7]} \mathrm{will} \mathrm{preserve} \mathrm{the} \mathrm{same} \mathrm{orderings} \mathrm{as}$ those in previously published papers that were constructed with different monotonic functions. Therefore, we decide to provide a reasonable explanation for researching the reason why a newer similarity measure will still derive the same results as those in already published papers. 
The rest of the paper is organized as follows. Section 2 of this paper introduced some previous results related to Ye and Fu [7] in terms of the tangent similarity measures and aggregation operators. Section 3 highlighted the theoretical results of Jaccard [126], Dice [127], and Salton and McGill [128]. Our theoretical development of two similarity measures using maximum norm, arithmetic mean, and two aggregation operators is presented in Section 4. Section 5 showed how this study used the subject example of $\mathrm{Ye}$ and $\mathrm{Fu}$ [7] as a comparison for the proposed two aggregation operators and thereby showing that it has the same diagnosis results. A detailed analysis is presented in Section 6 about the tangent and improved cosine similarity measures and how there are transformations of the proposed revision thereby showing that these complicated computations of $\mathrm{Ye} \mathrm{[6]} \mathrm{and} \mathrm{Ye}$ and $\mathrm{Fu}$ [7] are redundant. The discussion is concluded in Section 7.

\section{Review of Related Results for Ye and Fu [7]}

Wang et al. [4] defined an SVNS as $A=\left\{\left\langle x, T_{A}(x), I_{A}(x)\right.\right.$, $\left.\left.F_{A}(x)\right\rangle \mid x \in X\right\}$, such that $X$ is a universe of discourse with the three membership functions: truth $T_{A}(x)$, indeterminacy $I_{A}(x)$, and falsity $F_{A}(x)$ that satisfy $T_{A}(x), I_{A}(x)$, and $F_{A}(x)$ $\in[0,1]$.

For $A=\left\{\left\langle x_{j}, T_{A}\left(x_{j}\right), I_{A}\left(x_{j}\right), F_{A}\left(x_{j}\right)\right\rangle \mid x_{j} \in X\right\}$ and $B=$ $\left\{\left\langle x_{j}, T_{B}\left(x_{j}\right), I_{B}\left(x_{j}\right), F_{B}\left(x_{j}\right)\right\rangle \mid x_{j} \in X\right\} \quad$ which are different SVNSs with a universal set $X=\left\{x_{1}, x_{2}, \cdots, x_{n}\right\}$, Ye and Fu [7] reviewed three similarity measures: Jaccard [126], Dice [127], and Salton and McGill [128] as follows.
Jaccard [126] defined the "Jaccard index"

$$
S_{J}(A, B)=\frac{1}{n} \sum_{j=1}^{n} \frac{\Omega_{j}}{\Delta_{A j}+\Delta_{B j}-\Omega_{j}},
$$

where

$$
\begin{aligned}
& \Omega_{j}=T_{A}\left(x_{j}\right) T_{B}\left(x_{j}\right)+I_{A}\left(x_{j}\right) I_{B}\left(x_{j}\right)+F_{A}\left(x_{j}\right) F_{B}\left(x_{j}\right), \\
& \Delta_{A j}=T_{A}^{2}\left(x_{j}\right)+I_{A}^{2}\left(x_{j}\right)+F_{A}^{2}\left(x_{j}\right), \\
& \Delta_{B j}=T_{B}^{2}\left(x_{j}\right)+I_{B}^{2}\left(x_{j}\right)+F_{B}^{2}\left(x_{j}\right) .
\end{aligned}
$$

Dice [127] defined the "Dice similarity measure":

$$
S_{D}(A, B)=\frac{1}{n} \sum_{j=1}^{n} \frac{2 \Omega_{j}}{\Delta_{A j}+\Delta_{B j}} .
$$

Salton and McGill [128] presented that

$$
S_{C}(A, B)=\frac{1}{n} \sum_{j=1}^{n} \frac{\Omega_{j}}{\sqrt{\Delta_{A j} \Delta_{B j}}}
$$

which is based on the inner product of two vectors and then normalizes the result.

Next, Ye and Fu [7] claimed that Equation (11) has some disadvantages such that they presented two improved cosine similarity measures as follows in Ye [6]:

$$
\begin{aligned}
& C_{1}(A, B)=\frac{1}{n} \sum_{j=1}^{n} \cos \left[\pi \frac{\max \left\{\left|T_{A}\left(x_{j}\right)-T_{B}\left(x_{j}\right)\right|,\left|I_{A}\left(x_{j}\right)-I_{B}\left(x_{j}\right)\right|,\left|F_{A}\left(x_{j}\right)-F_{B}\left(x_{j}\right)\right|\right\}}{2}\right], \\
& C_{2}(A, B)=\frac{1}{n} \sum_{j=1}^{n} \cos \left[\pi \frac{\left\{\left|T_{A}\left(x_{j}\right)-T_{B}\left(x_{j}\right)\right|+\left|I_{A}\left(x_{j}\right)-I_{B}\left(x_{j}\right)\right|+\left|F_{A}\left(x_{j}\right)-F_{B}\left(x_{j}\right)\right|\right\}}{6}\right] .
\end{aligned}
$$

Using the concept of tangency, Ye and Fu [7] developed two new equations as follows:

$$
T_{1}(A, B)=1-\frac{1}{n} \sum_{j=1}^{n} \tan \left[\pi \frac{\max \left\{\left|T_{A}\left(x_{j}\right)-T_{B}\left(x_{j}\right)\right|,\left|I_{A}\left(x_{j}\right)-I_{B}\left(x_{j}\right)\right|,\left|F_{A}\left(x_{j}\right)-F_{B}\left(x_{j}\right)\right|\right\}}{4}\right],
$$

and

$$
T_{2}(A, B)=1-\frac{1}{n} \sum_{j=1}^{n} \tan \left[\pi \frac{\left\{\left|T_{A}\left(x_{j}\right)-T_{B}\left(x_{j}\right)\right|+\left|I_{A}\left(x_{j}\right)-I_{B}\left(x_{j}\right)\right|+\left|F_{A}\left(x_{j}\right)-F_{B}\left(x_{j}\right)\right|\right\}}{12}\right] .
$$


Finally, Ye and Fu [7] constructed two weighted measures that are still built on tangency:

$$
\begin{aligned}
& T_{w 1}(A, B)=1-\sum_{j=1}^{n} w_{j} \tan \left[\pi \frac{\max \left\{\left|T_{A}\left(x_{j}\right)-T_{B}\left(x_{j}\right)\right|,\left|I_{A}\left(x_{j}\right)-I_{B}\left(x_{j}\right)\right|,\left|F_{A}\left(x_{j}\right)-F_{B}\left(x_{j}\right)\right|\right\}}{4}\right], \\
& T_{w 2}(A, B)=1-\sum_{j=1}^{n} w_{j} \tan \left[\pi \frac{\left\{\left|T_{A}\left(x_{j}\right)-T_{B}\left(x_{j}\right)\right|+\left|I_{A}\left(x_{j}\right)-I_{B}\left(x_{j}\right)\right|+\left|F_{A}\left(x_{j}\right)-F_{B}\left(x_{j}\right)\right|\right\}}{12}\right] .
\end{aligned}
$$

During a multiple period diagnosis, Ye and Fu [7] used tangent and improved cosine measures to find out the most probable disease suffered by the patient. We recall the decision algorithm proposed by $\mathrm{Ye}$ and $\mathrm{Fu}$ [7]:

Assign $S=\left\{S_{1}, S_{2}, \cdots, S_{m}\right\}$ as the group of different symptoms, $T=\left\{t_{1}, t_{2}, \cdots, t_{q}\right\}$ as a series of multiple periods, and $D=\left\{D_{1}, D_{2}, \cdots, D_{n}\right\}$ as a group of possible diagnoses. Let the weight of period $t_{k}$ be denoted as $w\left(t_{k}\right)$ with $w\left(t_{k}\right)>0$ and $\sum_{k=1}^{q} w\left(t_{k}\right)=1$. For a patient $P$ with various symptoms, Ye and Fu [7] used $S_{j}\left(t_{k}\right)$ to denote the SVNS for this patient throughout the period $t_{k}$ for symptoms $S_{j}$ and $C_{i j}$, and to represent the SVNS for disease $D_{i}$ related to the symptom $S_{j}$. The algorithm is cited as follows:

Step 1. Ye and Fu [7] applied $T_{2}$ of Equation (15) or $C_{2}$ of Equation (13) to compute the similarity between $S_{j}\left(t_{k}\right)$ and $C_{i j}$ as $T_{2}\left(P, D_{i}, t_{k}\right)$ or $C_{2}\left(P, D_{i}, t_{k}\right)$.

Remark 1. Ye and Fu [7] used $T_{w i}\left(P, t_{k}\right)$ where the relation of the patient's disease $D_{i}$ and the period $t_{k}$ is not indicated clearly. Hence, the proposed change of the expression is from $T_{w i}\left(P, t_{k}\right)$ to $T_{2}\left(P, D_{i}, t_{k}\right)$.

Step 2. To derive the weighted aggregation value, $M\left(P, D_{i}\right)$ for the patient related to the disease $D_{i}$ :

$$
M_{T_{2}}\left(P, D_{i}\right)=\sum_{k=1}^{q} w\left(t_{k}\right) T_{2}\left(P, D_{i}, t_{k}\right)
$$

or

$$
M_{C_{2}}\left(P, D_{i}\right)=\sum_{k=1}^{q} w\left(t_{k}\right) C_{2}\left(P, D_{i}, t_{k}\right)
$$

Step 3. Present the most probable diagnosis to the patient according to the highest amount among weighted aggregations.

Step 4. Finish.

\section{Our Theoretical Results for Similarity Measures of Jaccard, Dice, and Cosine}

This section demonstrated that

$$
S_{C}(A, B) \geq S_{D}(A, B) \geq S_{J}(A, B)
$$

Hence, we will prove that relations among the three similarity measures proposed by Jaccard [126], Dice [127], and Ye [6].

Building on the concept of the arithmetic average is greater than or equal to the geometric average, it is found that

$$
\frac{\Delta_{A j}+\Delta_{B j}}{2} \geq \sqrt{\Delta_{A j} \Delta_{B j}},
$$

and thereby deriving that

$$
S_{C}(A, B) \geq S_{D}(A, B) .
$$

From the definition of $\Delta_{A j}, \Delta_{B j}$, and $\Omega_{j}$ of Equations (7)-(9), respectively, the following is obtained:

$$
\begin{aligned}
\Delta_{A j}+\Delta_{B j}-2 \Omega_{j}= & \left(T_{A}\left(x_{j}\right)-T_{A}\left(x_{j}\right)\right)^{2}+\left(I_{A}\left(x_{j}\right)-I_{A}\left(x_{j}\right)\right)^{2} \\
& +\left(F_{A}\left(x_{j}\right)-F_{A}\left(x_{j}\right)\right)^{2} \geq 0,
\end{aligned}
$$

such that

$$
\begin{aligned}
\frac{2}{\Delta_{A j}+\Delta_{B j}} & \geq \frac{1}{\Delta_{A j}+\Delta_{B j}-\Omega_{j}}, \\
S_{D}(A, B) & \geq S_{J}(A, B) .
\end{aligned}
$$

Theorem 2 below summarizes the findings.

Theorem 2. Using two SVNSs $A=\left\{\left\langle x_{j}, T_{A}\left(x_{j}\right), I_{A}\left(x_{j}\right), F_{A}\right.\right.$ $\left.\left.\left(x_{j}\right)\right\rangle \mid x_{j} \in X\right\}$ and $B=\left\{\left\langle x_{j}, T_{B}\left(x_{j}\right), I_{B}\left(x_{j}\right), F_{B}\left(x_{j}\right)\right\rangle \mid x_{j} \in X\right\}$ together with the universe of discourse $X=\left\{x_{1}, x_{2}, \cdots, x_{n}\right\}$, $S_{C}(A, B) \geq S_{D}(A, B) \geq S_{J}(A, B)$ is confirmed. 
TABle 1: (Reproduction of Table 5 of Ye and Fu [7]) Characteristic values related to the patients and their symptoms for three periods.

\begin{tabular}{ccccccc}
\hline & $t_{k}$ & $S_{1}$ (temperature) & $S_{2}$ (headache) & $S_{3}$ (stomach pain) & $S_{4}$ (cough) & $S_{5}$ (chest pain) \\
\hline & $t_{1}$ & $(0.8,0.6,0.5)$ & $(0.5,0.4,0.3)$ & $(0.2,0.1,0.3)$ & $(0.7,0.6,0.3)$ & $(0.4,0.3,0.2)$ \\
$P_{1}$ & $t_{2}$ & $(0.7,0.3,0.2)$ & $(0.6,0.3,0.2)$ & $(0.3,0.2,0.4)$ & $(0.6,0.5,0.2)$ & $(0.6,0.5,0.3)$ \\
& $t_{3}$ & $(0.5,0.2,0.4)$ & $(0.6,0.3,0.4)$ & $(0.3,0.3,0.5)$ & $(0.4,0.3,0.2)$ & $(0.6,0.4,0.4)$ \\
\hline & $t_{1}$ & $(0.6,0.6,0.1)$ & $(0.1,0.2,0.6)$ & $(0.3,0.2,0.8)$ & $(0.6,0.2,0.3)$ & $(0.2,0.3,0.7)$ \\
$P_{2}$ & $t_{2}$ & $(0.5,0.4,0.2)$ & $(0.2,0.2,0.6)$ & $(0.2,0.1,0.7)$ & $(0.8,0.3,0.1)$ & $(0.1,0.1,0.8)$ \\
& $t_{3}$ & $(0.8,0.3,0.1)$ & $(0.2,0.1,0.5)$ & $(0.1,0.1,0.9)$ & $(0.7,0.2,0.0)$ & $(0.1,0.1,0.8)$ \\
\hline & $t_{1}$ & $(0.3,0.1,0.2)$ & $(0.3,0.2,0.2)$ & $(0.7,0.6,0.7)$ & $(0.3,0.2,0.2)$ & $(0.4,0.4,0.3)$ \\
$P_{3}$ & $t_{2}$ & $(0.4,0.2,0.2)$ & $(0.5,0.1,0.3)$ & $(0.4,0.2,0.2)$ & $(0.5,0.3,0.3)$ & $(0.6,0.3,0.2)$ \\
& $t_{3}$ & $(0.8,0.7,0.6)$ & $(0.7,0.5,0.5)$ & $(0.4,0.1,0.1)$ & $(0.7,0.3,0.4)$ & $(0.7,0.4,0.5)$ \\
\hline & $t_{1}$ & $(0.2,0.1,0.7)$ & $(0.2,0.3,0.7)$ & $(0.2,0.2,0.7)$ & $(0.2,0.1,0.8)$ & $(0.8,0.2,0.1)$ \\
$P_{4}$ & $t_{2}$ & $(0.1,0.1,0.6)$ & $(0.1,0.2,0.8)$ & $(0.2,0.1,0.8)$ & $(0.3,0.0,0.9)$ & $(0.7,0.1,0.2)$ \\
& $t_{3}$ & $(0.1,0.1,0.8)$ & $(0.1,0.2,0.7)$ & $(0.3,0.1,0.8)$ & $(0.2,0.1,0.9)$ & $(0.9,0.1,0.1)$ \\
\hline
\end{tabular}

\section{Our Proposed Similarity Measures}

Using the two SVNSs A and B with a universal set $X=\left\{x_{1}\right.$, $\left.x_{2}, \cdots, x_{n}\right\}$ as above, it is assumed that:

$$
M_{w 1}(A, B)=1-\sum_{j=1}^{n} w_{j} \alpha_{j}
$$

with

$$
\begin{aligned}
\alpha_{j}= & \max \left\{\left|T_{A}\left(x_{j}\right)-T_{B}\left(x_{j}\right)\right|,\left|I_{A}\left(x_{j}\right)-I_{B}\left(x_{j}\right)\right|,\right. \\
& \left.\cdot\left|F_{A}\left(x_{j}\right)-F_{B}\left(x_{j}\right)\right|\right\},
\end{aligned}
$$

and

$$
M_{w 2}(A, B)=1-\sum_{j=1}^{n} w_{j} \beta_{j}
$$

with

$\beta_{j}=\frac{\left|T_{A}\left(x_{j}\right)-T_{B}\left(x_{j}\right)\right|+\left|I_{A}\left(x_{j}\right)-I_{B}\left(x_{j}\right)\right|+\left|F_{A}\left(x_{j}\right)-F_{B}\left(x_{j}\right)\right|}{3}$,

such that $M_{w 1}$ is related to the maximum norm, and $M_{w 2}$ is related to the arithmetic mean.

Our proposed weighted aggregation value for a patient, $P$, to a disease, $D$, is computed as

$$
M_{w 1}\left(P, D_{i}\right)=\sum_{k=1}^{q} w\left(t_{k}\right) M_{w 1}\left(S_{j}^{P}\left(t_{k}\right), C_{i j}\right),
$$

where $S_{j}^{P}\left(t_{k}\right)$ is the SVNS for the patient $P$, with the symptom $S_{j}$, at period $t_{k}$, and $C_{i j}$ is the SVNS for the disease $D_{i}$ and the symptom $S_{j}$. On the other hand, we provide a second aggregated value by our proposed second similarity measure of Equation (27) as

$$
M_{w 2}\left(P, D_{i}\right)=\sum_{k=1}^{q} w\left(t_{k}\right) M_{w 2}\left(S_{j}^{P}\left(t_{k}\right), C_{i j}\right) .
$$

\section{Diagnoses Illustration}

The example from Ye and $\mathrm{Fu}$ [7] is used, wherein a patient group presents with an array of diseases, $D=\left\{D_{1}, D_{2}, \cdots\right.$, $\left.D_{5}\right\}=\{$ viral fever, malaria, typhoid, gastritis, stenocardia $\}$, the list of symptoms as, $S=\left\{S_{1}, S_{2}, \cdots, S_{5}\right\}=\{$ temperature, headache, stomach pain, cough, chest pain\}, and a set of four patients, $\left\{P_{1}, P_{2}, P_{3}, P_{4}\right\}$. The characteristic values of the patients and symptoms for a certain period are listed in Table 1. For example, $S_{1}^{P_{1}}\left(t_{1}\right)$ is the SVNS for the patient $P_{1}$ with the symptom $S_{1}$ at period $t_{1}=(0.8,0.6,0.5)$.

Table 2 continues the list between (a) probable diseases and (b) their corresponding symptoms. For example, $C_{12}=(0.3,0.2,0.5)$ is the SVNS for disease $D_{1}$ with the symptom $S_{2}$.

With the proposed method of $M_{w 1}\left(P_{s}, D_{i}\right)$ and $M_{w 2}\left(P_{s}\right.$, $\left.D_{i}\right)$, for $s=1,2,3,4$ and $i=1,2, \cdots, 5$ with weights for periods $w\left(t_{1}\right)=0.25, w\left(t_{2}\right)=0.35$, and $w\left(t_{3}\right)=0.4$ and weights for symptoms $w_{j}=1 / 5$, for $j=1,2, \cdots, 5$, the results are presented in Table 3.

From Table 3, by the aggregation operator of $M_{w 1}$ with the maximum norm or the aggregation operator $M_{w 2}$ with the arithmetic mean, the same results are derived for patients $P_{1}$ and $P_{3}$ suffering from viral fever, the patient $P_{2}$ suffering malaria, and the patient $P_{4}$ suffering stenocardia. The study's derivations are the same as that of $\mathrm{Yu}$ and $\mathrm{Fu}$ [7] with the aggregation operator $M_{T_{2}}$ of Equation (18) and the aggregation operator $M_{C_{2}}$ of Equation (19). 
TABLE 2: (Reproduction of Table 4 of Ye and Fu [7]) Characteristic values related to five diseases and five symptoms.

\begin{tabular}{lccccc}
\hline & $S_{1}$ (temperature) & $S_{2}$ (headache) & $S_{3}$ (stomach pain) & $S_{4}$ (cough) & $S_{5}$ (chest pain) \\
\hline$D_{1}$ viral fever & $(0.4,0.6,0.0)$ & $(0.3,0.2,0.5)$ & $(0.1,0.3,0.7)$ & $(0.4,0.3,0.3)$ & $(0.1,0.2,0.7)$ \\
$D_{2}$ malaria & $(0.7,0.3,0.0)$ & $(0.2,0.2,0.6)$ & $(0.0,0.1,0.9)$ & $(0.7,0.3,0.0)$ & $(0.1,0.1,0.8)$ \\
$D_{3}$ typhoid & $(0.3,0.4,0.3)$ & $(0.6,0.3,0.1)$ & $(0.2,0.1,0.7)$ & $(0.2,0.2,0.6)$ & $(0.1,0.0,0.9)$ \\
$D_{4}$ gastritis & $(0.1,0.2,0.7)$ & $(0.2,0.4,0.4)$ & $(0.8,0.2,0.0)$ & $(0.2,0.1,0.7)$ & $(0.2,0.1,0.7)$ \\
$D_{5}$ stenocardia & $(0.1,0.1,0.8)$ & $(0.0,0.2,0.8)$ & $(0.2,0.0,0.8)$ & $(0.2,0.0,0.8)$ & $(0.8,0.1,0.1)$ \\
\hline
\end{tabular}

TABle 3: Study findings of $M_{w 1}\left(P_{s}, D_{i}\right)$ and $M_{w 2}\left(P_{s}, D_{i}\right)$.

\begin{tabular}{lcccccc}
\hline & $D_{1}$ & $D_{2}$ & $D_{3}$ & $D_{4}$ & $D_{5}$ & Diagnosis result \\
\hline$M_{w 1}\left(P_{1}, D_{i}\right)$ & $0.6730^{*}$ & 0.5990 & 0.6560 & 0.5260 & 0.5270 & $D_{1}$ viral fever \\
$M_{w 1}\left(P_{2}, D_{i}\right)$ & 0.7970 & $0.8730^{*}$ & 0.6780 & 0.5510 & 0.5330 & $D_{2}$ malaria \\
$M_{w 1}\left(P_{3}, D_{i}\right)$ & $0.5860^{*}$ & 05040 & 0.5540 & 0.5300 & 0.4520 & $D_{1}$ viral fever \\
$M_{w 1}\left(P_{4}, D_{i}\right)$ & 0.5210 & 0.4750 & 0.5640 & 0.6020 & $0.8910^{*}$ & $D_{5}$ stenocardia \\
$M_{w 2}\left(P_{1}, D_{i}\right)$ & $0.7770^{*}$ & 0.7323 & 0.7483 & 0.6810 & $0 / 6510$ & $D_{1}$ viral fever \\
$M_{w 2}\left(P_{2}, D_{i}\right)$ & 0.8683 & $0.9250^{*}$ & 0.7897 & 0.6883 & 0.6670 & $D_{2}$ malaria \\
$M_{w 2}\left(P_{3}, D_{i}\right)$ & $0.7573^{*}$ & 0.6983 & 0.6960 & 0.7133 & 0.6573 & $D_{1}$ viral fever \\
$M_{w 2}\left(P_{4}, D_{i}\right)$ & 0.6917 & 0.6617 & 0.7170 & 0.7577 & $0.9443^{*}$ & $D_{5}$ stenocardia \\
\hline
\end{tabular}

${ }^{*}$ Maximum values and thus, the most probable diagnosis.

\section{Further Discussion for Aggregation Operators}

In this section, we will explain that previous researchers used monotonic functions to transform the identity function (that is, $f(x)=x$ ) to complicated expressions. Based on the abbreviations of $\alpha_{j}$ and $\beta_{j}$, it is possible to rewrite $T_{w 1}$ of Equation (16) and $T_{w 2}$ of Equation (17) proposed by Yu and Fu [7] to simplify their expressions as follows

$$
\begin{aligned}
& T_{w 1}(A, B)=1-\sum_{j=1}^{n} w_{j} \tan \left(\pi \alpha_{j} / 4\right), \\
& T_{w 2}(A, B)=1-\sum_{j=1}^{n} w_{j} \tan \left(\pi \beta_{j} / 4\right) .
\end{aligned}
$$

Comparing Equation (25) with Equation (31), the following general expression is implied:

$$
\operatorname{Sim}_{w 1}(A, B)=1-\sum_{j=1}^{n} w_{j} f\left(\alpha_{j}\right)
$$

such that in Equation (25), for $M_{w 1}, f(x)=x$, and in Equation (31), for $T_{w 1}, f(x)=\tan (\pi x / 4)$.

By the same argument, comparing Equation (27) with Equation (32) obtains a general expression:

$$
\operatorname{Sim}_{w 2}(A, B)=1-\sum_{j=1}^{n} w_{j} f\left(\beta_{j}\right),
$$

such that in Equation (27), for $M_{w 2}, f(x)=x$, and in Equation (32), for $T_{w 2}, f(x)=\tan (\pi x / 4)$.

Consequently, we can further simplify $C_{1}(A, B)$ of Equation (12) and $C_{2}(A, B)$ of Equation (13) proposed by Ye [6] as follows

$$
\begin{aligned}
& C_{1}(A, B)=\frac{1}{n} \sum_{j=1}^{n} \cos \left(\pi \alpha_{j} / 2\right), \\
& C_{2}(A, B)=\frac{1}{n} \sum_{j=1}^{n} \cos \left(\pi \beta_{j} / 2\right) .
\end{aligned}
$$

Hence, Equations (25), (31), and (35) are used in comparison to determine their relationship with $\alpha_{j}$ of Equation (26). By the same observation, it is found that Equations (27), (32), and (36) are related to $\beta_{j}$ of Equation (28).

In Equations (25) and (27), the most natural approach $f(x)=x$ is applied by us. Meanwhile, in Equations (31) and (32), Ye and Fu [7] used $f(x)=\tan (\pi x / 4)$, and in Equations (35) and (36), Ye [6] used $f(x)=\cos (\pi x / 2)$.

$f(x)=x$ and $f(x)=\tan (\pi x / 4)$ are both increasing functions from $f(0)=0$ to $f(1)=1$. On the other hand, $f(x)=$ $\cos (\pi x / 2)$ is a decreasing function from $f(0)=1$ to $f(1)=$ 0 . All of them are monotonic functions.

For a similarity measure, say $\operatorname{Sim}, \operatorname{Sim}(0)=1$, and $\operatorname{Sim}(1)=0$ are ideal, such as in Equations (25), (27), (31), and (32) and then abstractly expressed in Equations (33) and (34), researchers use $1-\sum_{j=1}^{m} w_{j} f\left(\alpha_{j}\right)$ or $1-\sum_{j=1}^{m}$ $w_{j} f\left(\beta_{j}\right)$.

There are infinite increasing functions that satisfy $f(0)$ $=0$ and $f(1)=1$. However, Ye and Fu [7] did not provide 
any explanation of why $f(x)=\tan (\pi x / 4)$ was selected by them.

Moreover, there are infinite decreasing functions that satisfy $f(0)=1$ and $f(1)=0$. Ye [6] and Ye and Fu [7] also did not explain why $f(x)=\cos (\pi x / 2)$ was used.

Since our proposed approach to use the simplest form $f$ $(x)=x$ can still derive the desired diagnosis, the study recommends not to apply the complicated method proposed by Ye [6] with $f(x)=\cos (\pi x / 2)$ and Ye and Fu [7] with $f(x)=$ $\tan (\pi x / 4)$. Instead, using our simplest form $f(x)=x$ will help researchers solve their similarity measure problems and also reduce the complicated computation caused by $\mathrm{Ye}$ [6] and $\mathrm{Ye}$ and $\mathrm{Fu}[7]$.

For completeness, we point out that Section 5.3 of Ye and $\mathrm{Fu}$ [7] shows the comparative analysis of $\mathrm{Ye}$ and $\mathrm{Fu}$ [7]. It is mentioned that the multiple period method is better than a single period of Ye [6] since the latter poses more difficulty in giving a suitable diagnosis for a specific patient presenting with a specific disease. Recalling the example of Section 5 of $\mathrm{Ye}$ and $\mathrm{Fu}$ [7], it showed that the aggregation operator $M_{T_{2}}$ of Equation (18) was used with tangent similarity measure $T_{2}$ of Equation (15) and the aggregation operator $M_{C_{2}}$ of Equation (19) with tangent similarity measure $C_{2}$ of Equation (13) which was proposed by Ye [6]. Both operators derived the same diagnosis results for four patients as obtained in Table 3 by aggregation operator $M_{w 1}$ of Equation (29) and aggregation operator $M_{w 2}$ of Equation (30).

In $\mathrm{Ye}$ and $\mathrm{Fu}$ [7], the improved similarity measure $C_{2}$ of $\mathrm{Ye}$ [6] was used and repeated three times, then combined with the weighted mean such that the improved similarity measure $C_{2}$ of the latter study can be used to solve multiperiod medical diagnosis problems. Ye and $\mathrm{Fu}$ [7] already successfully applied $C_{2}$ in their algorithm as mentioned that using the tangent function and cosine measure, the diagnoses are similar and therefore proving that their proposed multiperiod method was indeed effective. The reference implies the possibility to use the cosine measure from Ye [6] for medical diagnoses over multiple periods. Ye and $\mathrm{Fu}$ [7] first used the improved cosine similarity measure of Equation (13) proposed by Ye [6] to support their tangent similarity measure of Equation (15), since aggregation approach by the two measures derive identical diagnosis results. On the contrary, in the same section, Ye and Fu [7] claimed that the improved cosine similarity measure of Equation (13) proposed by Ye [6] is unable to solve multiperiod medical diagnosis problems. Hence, the study points out that Section 5.2 or the comparative analysis of Ye and $\mathrm{Fu}$ [7] contained questionable results.

\section{Conclusion}

In this paper, we tried to claim that there is an isomorphism between (a) our simplest similarity measures and (b) those complicated similarity measures. Hence, to construct new similarity measures that can be explained by isomorphism is tedious and unnecessary. This study provided a detailed analysis of the improved cosine similarity measure proposed by Ye [6] and the tangent similarity measure proposed by Ye and $\mathrm{Fu}$ [7] to point out that these are transformations of the maximum norm and the arithmetic mean. As a recommendation, researchers can directly apply the well-known distance: the maximum norm and the arithmetic mean, to simplify the complicated computations in Ye and Fu [7].

\section{Conflicts of Interest}

The authors declare no conflict of interest.

\section{Acknowledgments}

This research was partially supported by the Ministry of Science and Technology, with grant no. MOST 107-2410H-241-001.

\section{References}

[1] L. A. Zadeh, "Fuzzy sets," Information and Control, vol. 8, pp. 338-353, 1995.

[2] F. Smarandache, A Unifying Field in Logics. Neutrosophy: Neutrosophic Probability, Set and Logic, American Research Press, Rehoboth, DE, 1999.

[3] H. Wang, F. Smarandache, Y. Q. Zhang, and R. Sunderraman, Interval Neutrosophic Sets and Logic: Theory and Applications in Computing, Hexis, Phoenix, AZ, 2005.

[4] H. Wang, F. Smarandache, Y. Q. Zhang, and R. Sunderraman, "Single valued neutrosophic sets," Multispace and Multistructure, vol. 4, pp. 410-413, 2010.

[5] J. Ye, "Multiple-attribute decision-making method under a single-valued neutrosophic hesitant fuzzy environment," Journal of Intelligent Systems, vol. 24, no. 1, pp. 23-36, 2015.

[6] J. Ye, "Improved cosine similarity measures of simplified neutrosophic sets for medical diagnoses," Artificial Intelligence in Medicine, vol. 63, no. 3, pp. 171-179, 2015.

[7] J. Ye and J. Fu, "Multi-period medical diagnosis method using a single valued neutrosophic similarity measure based on tangent function," Computer Methods and Programs in Biomedicine, vol. 123, pp. 142-149, 2016.

[8] X. Peng and J. Dai, "A bibliometric analysis of neutrosophic set: two decades review from 1998 to 2017," Artificial Intelligence Review, vol. 53, no. 1, pp. 199-255, 2020.

[9] J. Ye, "Generalized ordered weighted simplified neutrosophic cosine similarity measure for multiple attribute group decision making," International Journal of Cognitive Informatics and Natural Intelligence, vol. 14, no. 1, pp. 51-62, 2020.

[10] P. Biswas, S. Pramanik, and B. C. Giri, "Neutrosophic TOPSIS with group decision making," Studies in Fuzziness and Soft Computing, vol. 369, pp. 543-585, 2019.

[11] S. Broumi, A. Bakali, M. Talea et al., "Bipolar complex neutrosophic sets and its application in decision making problem," Studies in Fuzziness and Soft Computing, vol. 369, pp. 677-710, 2019.

[12] R. Chatterjee, P. Majumdar, and S. K. Samanta, "Similarity measures in neutrosophic sets-I," Studies in Fuzziness and Soft Computing, vol. 369, pp. 249-294, 2019.

[13] R. Chatterjee, P. Majumdar, and S. K. Samanta, "Similarity measures in neutrosophic sets-II," Studies in Fuzziness and Soft Computing, vol. 369, pp. 295-325, 2019. 
[14] W. Cui and J. Ye, "Logarithmic similarity measure of dynamic neutrosophic cubic sets and its application in medical diagnosis," Computers in Industry, vol. 111, pp. 198-206, 2019.

[15] C. Fan, "Correlation coefficients of refined-single valued neutrosophic sets and their applications in multiple attribute decision-making," Journal of Advanced Computational Intelligence and Intelligent Informatics, vol. 23, no. 3, pp. 421-426, 2019.

[16] C. Fan, S. Feng, and K. Hu, "Linguistic neutrosophic numbers einstein operator and its application in decision making," Mathematics, vol. 7, no. 5, p. 12, 2019.

[17] J. Fu, J. Ye, and W. Cui, “The Dice measure of cubic hesitant fuzzy sets and its initial evaluation method of benign prostatic hyperplasia symptoms," Scientific Reports, vol. 9, no. 1, p. 8, 2019.

[18] L. Gou and M. Wang, "Semantic risk analysis based on single-valued neutrosophic sets," IEEE Access, vol. 7, pp. 76480-76488, 2019.

[19] S. Jha, R. Kumar, L. H. Son et al., "Neutrosophic soft set decision making for stock trending analysis," Evolving Systems, vol. 10, no. 4, pp. 621-627, 2019.

[20] S. Jha, L. H. Son, R. Kumar, I. Priyadarshini, F. Smarandache, and H. V. Long, "Neutrosophic image segmentation with Dice coefficients," Measurement, vol. 134, pp. 762-772, 2019.

[21] G. Li, C. Niu, and C. Zhang, "Multi-criteria decision making approach using the fuzzy measures for environmental improvement under neutrosophic environment," Ekoloji, vol. 28, no. 107, pp. 1605-1615, 2019.

[22] H. Li, S. Zhang, J. Shi, and Y. Hu, "Research and design of intelligent learning system based on recommendation technology," Mechatronic Systems and Control, vol. 47, no. 1, pp. 43-49, 2019.

[23] Y. Li, J. Wang, and T. Wang, "A linguistic neutrosophic multi-criteria group decision-making approach with EDAS method," Arabian Journal for Science and Engineering, vol. 44, no. 3, pp. 2737-2749, 2019.

[24] C. Liu and Y. Luo, "New aggregation operators of singlevalued neutrosophic hesitant fuzzy set and their application in multi-attribute decision making," Pattern Analysis and Applications, vol. 22, no. 2, pp. 417-427, 2019.

[25] M. Luo, L. Wu, K. Zhou, and H. Zhang, "Multi-criteria decision making method based on the single valued neutrosophic sets1," Journal of Intelligent and Fuzzy Systems, vol. 37, no. 2, pp. 2403-2417, 2019.

[26] H. G. Nancy, "A novel divergence measure and its based TOPSIS method for multi criteria decision-making under single-valued neutrosophic environment," Journal of Intelligent \& Fuzzy Systems, vol. 36, no. 1, pp. 101-115, 2019.

[27] G. N. Nguyen, L. H. Son, A. S. Ashour, and N. Dey, "A survey of the state-of-the-arts on neutrosophic sets in biomedical diagnoses," International Journal of Machine Learning and Cybernetics, vol. 10, no. 1, p. 13, 2019.

[28] X. Peng, "New multiparametric similarity measure and distance measure for interval neutrosophic set with IoT industry evaluation," IEEE Access, vol. 7, pp. 28258-28280, 2019.

[29] X. Peng and J. Dai, "Interval neutrosophic reducible weighted Maclaurin symmetric means with internet of medical things (IoMt) industry evaluation," IEEE Access, vol. 7, pp. 6247962495, 2019.
[30] M. Rajangam and C. Annamalai, "Extractive document summarization using an adaptive, knowledge based cognitive model," Cognitive Systems Research, vol. 56, pp. 56-71, 2019.

[31] R. Şahin, "An approach to neutrosophic graph theory with applications," Soft Computing, vol. 23, no. 2, pp. 569-581, 2019.

[32] S. Shao, X. Zhang, and Q. Zhao, "Multi-attribute decision making based on probabilistic neutrosophic hesitant fuzzy Choquet aggregation operators," Symmetry, vol. 11, no. 5, p. 15, 2019.

[33] L. Shi and Y. Yuan, "Hybrid weighted arithmetic and geometric aggregation operator of neutrosophic cubic sets for MADM," Symmetry, vol. 11, no. 2, p. 10, 2019.

[34] C. Tian, W. Y. Zhang, S. Zhang, and J. J. Peng, “An extended single-valued neutrosophic projection-based qualitative flexible multi-criteria decision-making method," Mathematics, vol. 7, no. 1, p. 16, 2019.

[35] L. Wang, Q. Xia, H. Li, and Y. Cao, "Multi-criteria decision making method based on improved cosine similarity measure with interval neutrosophic sets," International Journal of Intelligent Computing and Cybernetics, vol. 12, no. 3, pp. 414-423, 2019.

[36] G. Wei and Z. Zhang, "Some single-valued neutrosophic Bonferroni power aggregation operators in multiple attribute decision making," Journal of Ambient Intelligence and Humanized Computing, vol. 10, no. 3, pp. 863-882, 2019.

[37] L. Xie, J. He, P. Cheng, R. Xiao, and X. Zhou, "A multi-criteria 2-tuple linguistic group decision-making method based on TODIM for cholecystitis treatments selection," IEEE Access, vol. 7, pp. 127967-127986, 2019.

[38] J. Ye, "PID tuning method using single-valued neutrosophic cosine measure and genetic algorithm," Intelligent Automation and Soft Computing, vol. 25, no. 1, pp. 15-23, 2019.

[39] Z. Zhai, J. M. Ortega, P. Castillejo, and V. Beltran, “A triangular similarity measure for case retrieval in CBR and its application to an agricultural decision support system," Sensors, vol. 19, no. 21, p. 23, 2019.

[40] Y. Zhang, L. Wang, X. Yu, and C. Yao, “A new concept of cosine similarity measures based on dual hesitant fuzzy sets and its possible applications," Cluster Computing, vol. 22, no. S6, pp. 15483-15492, 2019.

[41] S. Zhao, D. Wang, L. Changyong, and W. Lu, "Induced Choquet integral aggregation operators with single-valued neutrosophic uncertain linguistic numbers and their application in multiple attribute group decision-making," Mathematical Problems in Engineering, vol. 2019, 14 pages, 2019.

[42] M. Akram, M. Nasir, and K. P. Shum, "Novel applications of bipolar single-valued neutrosophic competition graphs," Applied Mathematics, vol. 33, no. 4, pp. 436-467, 2018.

[43] M. Ali, L. H. Son, N. D. Thanh, and N. V. Minh, "A neutrosophic recommender system for medical diagnosis based on algebraic neutrosophic measures," Applied Soft Computing Journal, vol. 71, pp. 1054-1071, 2018.

[44] T. Cai and X. Yang, "Non-structured data integration access policy using hadoop," Wireless Personal Communications, vol. 102, no. 2, pp. 895-908, 2018.

[45] C. Fan, E. Fan, and J. Ye, "The cosine measure of singlevalued neutrosophic multisets for multiple attribute decision-making," Symmetry, vol. 10, no. 5, p. 13, 2018.

[46] N. H. Garg, "Non-linear programming method for multicriteria decision making problems under interval 
neutrosophic set environment," Applied Intelligence, vol. 48, no. 8, pp. 2199-2213, 2018.

[47] H. Guan, J. He, A. Zhao, Z. Dai, and S. Guan, “A forecasting model based on multi-valued neutrosophic sets and two-factor, third-order fuzzy fluctuation logical relationships," Symmetry, vol. 10, no. 7, p. 18, 2018.

[48] F. Karaaslan and K. Hayat, "Some new operations on singlevalued neutrosophic matrices and their applications in multicriteria group decision making," Applied Intelligence, vol. 48, no. 12, pp. 4594-4614, 2018.

[49] Q. Khan, P. Liu, and T. Mahmood, "Some generalized Dice measures for double-valued neutrosophic sets and their applications," Mathematics, vol. 6, no. 7, p. 16, 2018.

[50] G. D. Küçük and R. Şahin, “A novel hybrid approach for simplified neutrosophic decision-making with completely unknown weight information," International Journal for Uncertainty Quantification, vol. 8, no. 2, pp. 161-173, 2018.

[51] C. Liu, "New similarity measures of simplified neutrosophic sets and their applications," Journal of Information Processing Systems, vol. 14, no. 3, pp. 790-800, 2018.

[52] D. Liu, G. Liu, and Z. Liu, "Some similarity measures of neutrosophic sets based on the euclidean distance and their application in medical diagnosis," Computational and Mathematical Methods in Medicine, vol. 2018, 9 pages, 2018.

[53] P. Liu, T. Mahmood, and Q. Khan, “Group decision making based on power heronian aggregation operators under linguistic neutrosophic environment," International Journal of Fuzzy Systems, vol. 20, no. 3, pp. 970-985, 2018.

[54] P. Liu and X. You, "Some Maclaurin symmetric mean operators based on neutrosophic linguistic numbers for multiattribute group decision making," Informatica, vol. 29, no. 4, pp. 711-732, 2018.

[55] K. Mondal, S. Pramanik, and B. C. Giri, "Hybrid binary logarithm similarity measure for MAGDM problems under SVNS assessments," Neutrosophic Sets and Systems, vol. 20, pp. 12-25, 2018.

[56] K. Mondal, S. Pramanik, and B. C. Giri, "Interval neutrosophic tangent similarity measure based MADM strategy and its application to MADM problems," Neutrosophic Sets and Systems, vol. 19, pp. 47-56, 2018.

[57] K. Mondal, S. Pramanik, and B. C. Giri, "Single valued neutrosophic hyperbolic sine similarity measure based MADM strategy," Neutrosophic Sets and Systems, vol. 20, pp. 3-11, 2018.

[58] R. T. Ngan, M. Ali, and L. H. Son, " $\delta$-Equality of intuitionistic fuzzy sets: a new proximity measure and applications in medical diagnosis," Applied Intelligence, vol. 48, no. 2, pp. 499525, 2018.

[59] H. Peng, H. Zhang, and J. Wang, "Probability multi-valued neutrosophic sets and its application in multi-criteria group decision-making problems," Neural Computing and Applications, vol. 30, no. 2, pp. 563-583, 2018.

[60] S. Pramanik, S. Dalapati, S. Alam, and T. K. Roy, "NCVIKOR based MAGDM strategy under neutrosophic cubic set environment," Neutrosophic Sets and Systems, vol. 20, pp. 95-108, 2018.

[61] S. Pramanik, R. Mallick, and A. Dasgupta, "Contributions of selected Indian researchers to multi attribute decision making in neutrosophic environment: an overview," Neutrosophic Sets and Systems, vol. 20, pp. 109-131, 2018.
[62] R. Şahin, "Normal neutrosophic multiple attribute decision making based on generalized prioritized aggregation operators," Neural Computing and Applications, vol. 30, no. 10, pp. 3095-3115, 2018.

[63] P. K. Singh, "Medical diagnoses using three-way fuzzy concept lattice and their Euclidean distance," Computational and Applied Mathematics, vol. 37, no. 3, pp. 3283-3306, 2018.

[64] Z. Tian, J. Wang, H. Zhang, and J. Wang, "Multi-criteria decision-making based on generalized prioritized aggregation operators under simplified neutrosophic uncertain linguistic environment," International Journal of Machine Learning and Cybernetics, vol. 9, no. 3, pp. 523-539, 2018.

[65] A. Tu, J. Ye, and B. Wang, "Multiple attribute decisionmaking method using similarity measures of neutrosophic cubic sets," Symmetry, vol. 10, no. 6, p. 11, 2018.

[66] G. Wei and Y. Wei, "Some single-valued neutrosophic dombi prioritized weighted aggregation operators in multiple attribute decision making," Journal of Intelligent and Fuzzy Systems, vol. 35, no. 2, pp. 2001-2013, 2018.

[67] H. Wu, Y. Yuan, L. Wei, and L. Pei, “On entropy, similarity measure and cross-entropy of single-valued neutrosophic sets and their application in multi-attribute decision making," Soft Computing, vol. 22, no. 22, pp. 7367-7376, 2018.

[68] Q. Wu, P. Wu, L. Zhou, H. Chen, and X. Guan, "Some new Hamacher aggregation operators under single-valued neutrosophic 2-tuple linguistic environment and their applications to multi-attribute group decision making," Computers and Industrial Engineering, vol. 116, pp. 144-162, 2018.

[69] W. Xiong and J. Cheng, "A novel method for determining the attribute weights in the multiple attribute decision-making with neutrosophic information through maximizing the generalized single-valued neutrosophic deviation," Information, vol. 9, no. 6, p. 14, 2018.

[70] J. Ye, "Operations and aggregation method of neutrosophic cubic numbers for multiple attribute decision-making," Soft Computing, vol. 22, no. 22, pp. 7435-7444, 2018.

[71] L. Zeming, G. Jianmin, J. Hongquan, G. Xu, G. Zhiyong, and W. Rongxi, "A similarity-based method for remaining useful life prediction based on operational reliability," Applied Intelligence, vol. 48, no. 9, pp. 2983-2995, 2018.

[72] X. Zhang, C. Bo, F. Smarandache, and J. Dai, "New inclusion relation of neutrosophic sets with applications and related lattice structure," International Journal of Machine Learning and Cybernetics, vol. 9, no. 10, pp. 1753-1763, 2018.

[73] J. Chen and J. Ye, "Some single-valued neutrosophic dombi weighted aggregation operators for multiple attribute decision-making," Symmetry, vol. 9, no. 6, p. 11, 2017.

[74] I. Deli and Y. Şubaş, "Some weighted geometric operators with SVTrN-numbers and their application to multi-criteria decision making problems," Journal of Intelligent and Fuzzy Systems, vol. 32, no. 1, pp. 291-301, 2017.

[75] C. Fan and J. Ye, "The cosine measure of refined-single valued neutrosophic sets and refined-interval neutrosophic sets for multiple attribute decision-making," Journal of Intelligent and Fuzzy Systems, vol. 33, no. 4, pp. 22812289, 2017.

[76] J. Fu and J. Ye, "Simplified neutrosophic exponential similarity measures for the initial evaluation/diagnosis of benign prostatic hyperplasia symptoms," Symmetry, vol. 9, no. 8, p. 10, 2017. 
[77] J. Hu, J. Pan, and X. Chen, "An interval neutrosophic projection-based VIKOR method for selecting doctors," Cognitive Computation, vol. 9, no. 6, pp. 801-816, 2017.

[78] Z. Lu and J. Ye, "Cosine measures of neutrosophic cubic sets for multiple attribute decision-making," Symmetry, vol. 9, no. 7, p. 10, 2017.

[79] Y. Ma, J. Wang, and X. Wu, "An interval neutrosophic linguistic multi-criteria group decision-making method and its application in selecting medical treatment options," Neural Computing and Applications, vol. 28, no. 9, pp. 2745-2765, 2017.

[80] J. Peng, J. Wang, and X. Wu, "An extension of the ELECTRE approach with multi-valued neutrosophic information," Neural Computing and Applications, vol. 28, no. S1, pp. 1011-1022, 2017.

[81] J. Peng, J. Wang, L. Yang, and J. Qian, “A novel multi-criteria group decision-making approach using simplified neutrosophic information," International Journal for Uncertainty Quantification, vol. 7, no. 4, pp. 355-376, 2017.

[82] J. Peng, J. Wang, and W. Yang, "A multi-valued neutrosophic qualitative flexible approach based on likelihood for multicriteria decision-making problems," International Journal of Systems Science, vol. 48, no. 2, pp. 425-435, 2016.

[83] S. Pramanik, P. Biswas, and B. C. Giri, "Hybrid vector similarity measures and their applications to multi-attribute decision making under neutrosophic environment," Neural Computing and Applications, vol. 28, no. 5, pp. 1163-1176, 2017.

[84] P. K. Singh, "Complex vague set based concept lattice," Chaos, Solitons and Fractals, vol. 96, pp. 145-153, 2017.

[85] N. D. Thanh, M. Ali, and L. H. Son, "A novel clustering algorithm in a neutrosophic recommender system for medical diagnosis," Cognitive Computation, vol. 9, no. 4, pp. 526544, 2017.

[86] Z. Tian, J. Wang, J. Wang, and H. Zhang, "An improved MULTIMOORA approach for multi-criteria decisionmaking based on interdependent inputs of simplified neutrosophic linguistic information," Neural Computing and Applications, vol. 28, no. S1, pp. 585-597, 2017.

[87] N. Wang and H. Zhang, "Probability multivalued linguistic neutrosophic sets for multi-criteria group decision-making," International Journal for Uncertainty Quantification, vol. 7, no. 3, pp. 207-228, 2017.

[88] D. Xu, C. Wei, and G. Wei, "TODIM method for singlevalued neutrosophic multiple attribute decision making," Information, vol. 8, no. 4, p. 18, 2017.

[89] Y. Zhou and M. Abdel Wahab, "Cosine based and extended transmissibility damage indicators for structural damage detection," Engineering Structures, vol. 141, pp. 175-183, 2017.

[90] C. C. Chou, "A generalized similarity measure for fuzzy numbers," Journal of Intelligent and Fuzzy Systems, vol. 30, no. 2, pp. 1147-1155, 2016.

[91] P. Liu and G. Tang, "Multi-criteria group decision-making based on interval neutrosophic uncertain linguistic variables and Choquet integral," Cognitive Computation, vol. 8, no. 6, pp. 1036-1056, 2016.

[92] F. Meng, X. Su, and Z. Qu, "Nonlinear approach for estimating WCET during programming phase," Cluster Computing, vol. 19, no. 3, pp. 1449-1459, 2016.

[93] N. H. Garg, "Novel single-valued neutrosophic aggregated operators under frank norm operation and its application to decision-making process," International Journal for Uncertainty Quantification, vol. 6, no. 4, pp. 361-375, 2016.

[94] L. Shi, "Correlation coefficient of simplified neutrosophic sets for bearing fault diagnosis," Shock and Vibration, vol. 2016, 11 pages, 2016.

[95] L. H. Son, "Generalized picture distance measure and applications to picture fuzzy clustering," Applied Soft Computing Journal, vol. 46, pp. 284-295, 2016.

[96] J. Ye, "Multiple-attribute group decision-making method under a neutrosophic number environment," Journal of Intelligent Systems, vol. 25, no. 3, pp. 377-386, 2016.

[97] H. Zhang, P. Ji, J. Wang, and X. Chen, "A neutrosophic normal cloud and its application in decision-making," Cognitive Computation, vol. 8, no. 4, pp. 649-669, 2016.

[98] C. Zhang, D. Li, and Y. Yan, “A Dual Hesitant Fuzzy Multigranulation Rough Set over Two-Universe Model for Medical Diagnoses," Computational and Mathematical Methods in Medicine, vol. 2015, 12 pages, 2015.

[99] M. Zhang, H. Hu, Z. He, L. Gao, and L. Sun, "Efficient linkbased similarity search in web networks," Expert Systems with Applications, vol. 42, no. 22, pp. 8868-8880, 2015.

[100] P. Liu, Q. Khan, and T. Mahmood, "Group decision making based on power heronian aggregation operators under neutrosophic cubic environment," Soft Computing, vol. 24, no. 3, pp. 1971-1997, 2020.

[101] A. Awang, N. A. H. Aizam, and L. Abdullah, “An integrated decision-making method based on neutrosophic numbers for investigating factors of coastal erosion," Symmetry, vol. 11, no. 3, p. 26, 2019.

[102] K. Hu, W. He, J. Ye, L. Zhao, H. Peng, and J. Pi, “Online visual tracking of weighted multiple instance learning via neutrosophic similarity-based objectness estimation," Symmetry, vol. 11 , no. 6 , p. $24,2019$.

[103] H. V. Long, M. Ali, H. Son, M. Khan, and D. N. Tu, “A novel approach for fuzzy clustering based on neutrosophic association matrix," Computers and Industrial Engineering, vol. 127, pp. 687-697, 2019.

[104] H. Ren, S. Xiao, and H. Zhou, "A chi-square distance-based similarity measure of single-valued neutrosophic set and applications," International Journal of Computers, Communications and Control, vol. 14, no. 1, pp. 78-89, 2019.

[105] K. Shen, X. Wang, and J. Wang, "Multi-criteria decisionmaking method based on smallest enclosing circle in incompletely reliable information environment," Computers and Industrial Engineering, vol. 130, pp. 1-13, 2019.

[106] P. Smith, "Exploring public transport sustainability with neutrosophic logic," Transportation Planning and Technology, vol. 42, no. 3, pp. 257-273, 2019.

[107] M. Akram, S. Shahzadi, and F. Smarandache, "Multi-attribute decision-making method based on neutrosophic soft rough information," Axioms, vol. 7, no. 1, p. 24, 2018.

[108] K. Hu, E. Fan, J. Ye, J. Pi, L. Zhao, and S. Shen, "Elementweighted neutrosophic correlation coefficient and its application in improving CAMShift tracker in RGBD video," Information, vol. 9, no. 5, p. 16, 2018.

[109] P. Ji, J. Wang, and H. Zhang, "Frank prioritized bonferroni mean operator with single-valued neutrosophic sets and its application in selecting third-party logistics providers," $\mathrm{Neu}$ ral Computing and Applications, vol. 30, no. 3, pp. 799-823, 2018. 
[110] P. Ji, H. Zhang, and J. Wang, "A projection-based TODIM method under multi-valued neutrosophic environments and its application in personnel selection," Neural Computing and Applications, vol. 29, no. 1, pp. 221-234, 2018.

[111] F. Jin, Z. Ni, H. Chen, R. Langari, X. Zhu, and H. Yuan, "Single-valued neutrosophic entropy and similarity measures to solve supplier selection problems," Journal of Intelligent and Fuzzy Systems, vol. 35, no. 6, pp. 6513-6523, 2018.

[112] F. Karaaslan, "Multicriteria decision-making method based on similarity measures under single-valued neutrosophic refined and interval neutrosophic refined environments," International Journal of Intelligent Systems, vol. 33, no. 5, pp. 928-952, 2018.

[113] M. Khan, L. H. Son, M. Ali, H. T. M. Chau, N. T. N. Na, and F. Smarandache, "Systematic review of decision making algorithms in extended neutrosophic sets," Symmetry, vol. 10, no. 8, p. 26, 2018.

[114] S. Naz, M. Akram, and F. Smarandache, "Certain notions of energy in single-valued neutrosophic graphs," Axioms, vol. 7, no. 3, p. 30, 2018.

[115] X. Peng and J. Dai, "Approaches to single-valued neutrosophic MADM based on MABAC, TOPSIS and new similarity measure with score function," Neural Computing and Applications, vol. 29, no. 10, pp. 939-954, 2018.

[116] V. Uluçay, I. Deli, and M. Şahin, "Similarity measures of bipolar neutrosophic sets and their application to multiple criteria decision making," Neural Computing and Applications, vol. 29, no. 3, pp. 739-748, 2018.

[117] S. Ashraf, S. Naz, H. Rashmanlou, and M. A. Malik, "Regularity of graphs in single valued neutrosophic environment," Journal of Intelligent and Fuzzy Systems, vol. 33, no. 1, pp. 529-542, 2017.

[118] H. Guan, S. Guan, and A. Zhao, "Forecasting model based on neutrosophic logical relationship and Jaccard similarity," Symmetry, vol. 9, no. 9, p. 16, 2017.

[119] Z. Guo, Y. Liu, and H. Yang, "A novel rough set model in generalized single valued neutrosophic approximation spaces and its application," Symmetry, vol. 9, no. 7, p. 19, 2017.

[120] S. Naz, H. Rashmanlou, and M. A. Malik, "Operations on single valued neutrosophic graphs with application," Journal of Intelligent and Fuzzy Systems, vol. 32, no. 3, pp. 2137-2151, 2017.

[121] X. Peng and J. Dai, “Algorithms for interval neutrosophic multiple attribute decision-making based on mabac, similarity measure, and EDAS," International Journal for Uncertainty Quantification, vol. 7, no. 5, pp. 395-421, 2017.

[122] P. Julian, K. C. Hung, and S. J. Lin, "On the Mitchell similarity measure and its application to pattern recognition," Pattern Recognition Letters, vol. 33, no. 9, pp. 1219-1223, 2012.

[123] H. B. Mitchell, "On the Dengfeng-Chuntian similarity measure and its application to pattern recognition," Pattern Recognition Letters, vol. 24, no. 16, pp. 3101-3104, 2003.

[124] P. S. Deng and H. C. J. Chao, "Analysis on comparison of distances derived by one-norm and two-norm with weight functions," Applied Mathematics and Computation, vol. 219, no. 17, pp. 9093-9098, 2013.

[125] C. Tung, S. Liu, and B. S. Wang, "A comment on "On the Mitchell similarity measure and its application to pattern recognition"," Pattern Recognition Letters, vol. 34, no. 5, pp. 453-455, 2013.
[126] P. Jaccard, "Distribution de la flore alpine dans le Bassin des Drouces et dans quelques regions voisines," Bulletin de la Société Vaudoise des Sciences Naturelles, vol. 37, no. 140, pp. 241-272, 1901.

[127] L. R. Dice, "Measures of the amount of Ecologic Association between Species," Ecology, vol. 26, no. 3, pp. 297-302, 1945.

[128] G. Salton and M. J. McGill, Introduction to Modern Information Retrieval, McGraw-Hill, New York, NY, USA, 1987. 